\section{Kalkhoden}

— Ein 32-jähriger Mann suchte die Nothilfe wegen Dyspnoe und Hämoptysen auf. Bei der körperlichen Untersuchung stellte man als Zufallsbefund weißlichgelbe Knötchen in der Skrotalhaut fest (Abbildung A). Die indolenten derben Knötchen erlaubten die klinische Blickdiagnose einer Calcinosis scrotalis.

Im Kontrastmittel-CT des Thorax und des Abdomens fanden sich zahlreiche kleine Lungenemboli, woraufhin man eine Antikoagulation begann. Im Becken-CT stellten sich die intrakutan
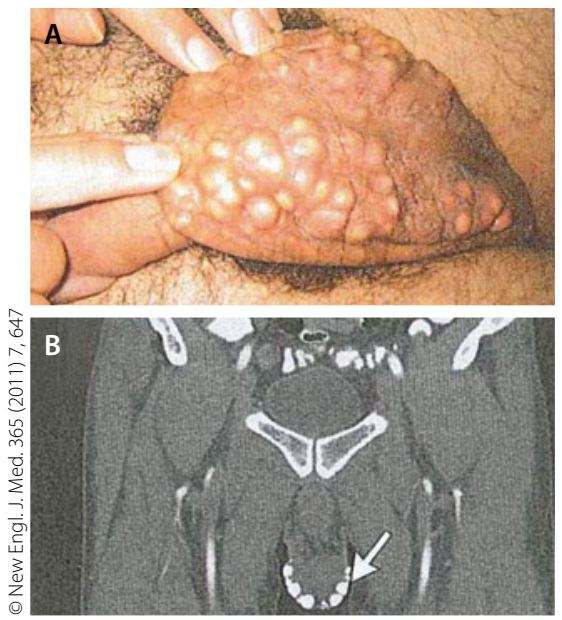

Calcinosis scrotalis: klinisches Bild (A) und $\mathrm{CT}(\mathrm{B})$.

liegenden verkalkten Knoten der Skrotalhaut in einer Größe von 3-8 $\mathrm{mm}$ dar (Abbildung B, Pfeil).

\section{Kommentar}

Bei der Calcinosis scrotalis handelt es sich um eine gutartige Veränderung ungeklärter Ätiopathogenese. Störungen des Kalziumoder Phosphatstoffwechsels liegen nicht vor. Die intradermal gelegenen Knoten nehmen meist im Laufe von Jahren an Größe zu und können exulzerieren, sodass sich weißliches, kreideartiges Material entleert. Die meisten Patienten wollen die Knoten aus ästhetischen Gründen entfernt haben.

H. S. FÜESSL

- T. Grenader, L. Shavit

(Shaare Zedec Medical Center, Jerusalem, Israel; talgrenader65@hotmail.com): Scrotal calcinosis. New Engl. J. Med. 365 (2011) 7, 647

\title{
Hohes kardiovaskuläre Risiko - schneller geistiger Abbau
}

\section{Ein erhöhtes kardiovaskuläres Risiko im mittleren Lebensalter geht nach einer aktuellen Studie mit einer ver- minderten kognitiven Leistungsfähig- keit und teilweise auch mit einem verstärkten kognitiven Abbau einher.}

- In einer Auswertung der britischen Whitehall-II-Studie wurden Daten von 3486 Männern und 1314 Frauen herangezogen, die bei Studienbeginn im Durchschnitt 55 Jahre alt waren und keine kardiovaskulären Erkrankungen hatten. Das kardiovaskuläre Risiko bei Studienbeginn wurde mit dem Framingham General Cardiovascular Risk Profile (FGCRP) ermittelt, das die Faktoren Alter, Geschlecht, HDL- und Gesamtcholesterin, systolischer Blutdruck, Diabetes und Rauchen berücksichtigt. Die kognitive Leistungsfähigkeit wurde bei Studienbeginn sowie im Follow up nach fünf und zehn Jahren untersucht, wobei die Funktionsbereiche logisches Denken, Gedächtnis, Wortflüssigkeit und Wortschatz erfasst wurden.

In der Querschnittsanalyse war ein höheres kardiovaskuläres Risiko schon bei Studienbeginn mit einer geringeren globalen kognitiven Leistungsfähigkeit und mit geringeren Leistungen in allen einzelnen Funktionsbereichen assoziiert. Dieser Zusammenhang fand sich bei Männern und Frauen und blieb weitgehend bestehen, wenn in multivariaten Analysen für Alter, Familienstand und Bildung adjustiert wurde. Darüber hinaus war bei Männern in der Längsschnittanalyse ein höheres kardiovaskulären Risiko auch mit einem stärkeren kognitiven Abbau während des Follow up verbunden, insbesondere im Bereich logisches Denken.

Wenn zusätzlich für die antihypertensive Therapie adjustiert wurde, verringerte sich der negative Einfluss des kardiovaskulären Risikos auf die kognitive Leistungsfähigkeit.

\section{Kommentar}

Angesichts des demografischen Wandels mit kontinuierlicher Zunahme des Anteils älterer Menschen in der Bevölkerung und Anstieg der individuellen Lebenserwartung gewinnen Demenzerkrankungen und kognitive Einschränkungen im Alter zunehmend an Bedeutung. Da die verfügbaren Therapien keine Heilung von Demenzerkrankungen ermöglichen und deren Krankheitsverlauf nur geringfügig beeinflussen, hat die Erforschung von Ansatzpunkten für Präventivmaßnahmen einen hohen Stellenwert. Die vorliegende Studie bestätigt und ergänzt frühere Studienergebnisse und weist einmal mehr darauf hin, dass kardiovaskuläre Risikofaktoren wahrscheinlich auch eine wichtige Bedeutung für demenzielle Erkrankungen haben und mögliche Ansatzpunkte für deren Prävention darstellen.

Randomisierte Therapiestudien mit einem sehr langen Follow up sind nötig, um zu untersuchen, ob eine frühzeitige Behandlung kardiovaskulärer Risikofaktoren im mittleren Lebensalter einen langfristigen Einfluss auf die kognitive Gesundheit im höheren Alter hat. Bis zum Vorliegen solcher Studien können betroffene Patienten schon jetzt über den Einfluss des vaskulären Risikoprofils auf die kognitive Leistungsfähigkeit aufgeklärt werden, um die Therapieadhärenz und die Bereitschaft zur Lebensstiländerung zu fördern.

DR. MED. MARKUS BUSCH, ROBERT KOCH-INSTITUT, BERLIN =

\footnotetext{
- Kaffashian et al.

Predictive utility of the Framingham general cardiovascular disease risk profile for cognitive function: evidence from the Whitehall II study. Eur. Heart J. 2011 (E-pub ahead of print)
} 